\title{
Sociedad civil en América Latina: Una conversación con el Dr. Alberto J. Olvera
}

\author{
ANDRÉs Roldán ToNioni \\ Universidad de Chile, Chile
}

RESUMEN Se presenta a continuación una entrevista al Profesor Alberto J. Olvera, destacado investigador de los procesos de construcción democrática en México y en Latinoamérica, realizada en la Facultad de Ciencias Sociales de la Universidad de Chile ${ }^{1}$. En esta conversación se intentó profundizar en torno a la noción de sociedad civil y sus comprensiones actuales.

El entendimiento de la sociedad civil en los contextos postautoritarios ha llevado a replantear la noción en base a las experiencias y a las transformaciones de las agrupaciones sociales que la componen. Este concepto ha adquirido un lugar importante en la teoría política y para el caso de Latino América sirvió para dar cuenta de la organización y resistencia de la sociedad durante las dictaduras militares. Revisamos en esta entrevista la evolución de esta categoría analítica y su funcionamiento para interpretar la sociedad civil en los actuales regímenes democráticos.

PALABRAS CLAVE Sociedad civil, Democratización, Conflicto, Estado.

ABSTRACT The following is an interview with Professor Alberto J. Olvera, an outstanding researcher who works on processes of democratic construction in Mexico and in Latin America, held at the Faculty of Social Sciences of the University of Chile. In this conversation, we tried to deepen into the notion of civil society and its current understandings.

The understanding of civil society in post-authoritarian contexts has led to rethinking the notion based on the experiences and the transformations of the social groups that compose it. This concept has gained an important place in political theory and in Latin America it served to account for the organization and resistance of society during military dictatorships.

1. Esta entrevista se desarrolló en el marco de la visita del profesor Olvera al Programa de Doctorado en Ciencias Sociales de la Universidad de Chile, en agosto de 2017. 
In this interview we review the evolution of this analytical category and its utility to interpret civil society in the current democratic regimes.

KEYWORDS Civil society, Democratization, Conflict, State.

\section{Introducción}

El profesor Alberto J. Olvera es Doctor en Sociología por la New School for Social Research de Nueva York e investigador del Instituto de Investigaciones HistóricoSociales de la Universidad Veracruzana. Es miembro del Sistema Nacional de Investigadores (SNI), Nivel III, y de la Academia Mexicana de Ciencias. Sus temas de investigación son: sociedad civil y construcción democrática, participación política y movimientos sociales en México y América Latina. Algunos de sus libros principales son La Sociedad Civil: de la Teoría a la Realidad (1999); Sociedad Civil, Esfera Pública y Democratización en América Latina: México (2003); con Ernesto Isunza, Democratización, Rendición de Cuentas y Sociedad Civil: participación ciudadana y control social (2006); con Evelina Dagnino y Aldo Panfichi, La Disputa por la Construcción Democrática en América Latina (2006, 2010). Su libro más reciente sobre el tema, como coordinador, es La Democratización Frustrada: Limitaciones Institucionales y Colonización Politica de las Instituciones Garantes de Derechos y de Participación Ciudadana en México (2010). Tiene más de 100 capítulos de libros y artículos en revistas publicados a lo largo de su carrera. Asimismo, ha participado de iniciativas de la sociedad civil mexicana, como experto en comisiones nacionales, en grupos de estudios en temáticas afines y como editorialista en diversos medios nacionales.

El uso del concepto de sociedad civil ha tenido diversas interpretaciones en la historia del pensamiento social y político, lo que ha generado debates acerca de su alcance y sentido, dependiendo de la tradición teórica desde la cual se utilice. No obstante estas diferencias, que van desde las lecturas liberales hasta las interpretaciones marxistas, la noción de sociedad civil pretende dar cuenta del complejo tejido social que soporta la participación de las personas en las organizaciones e instituciones que trascienden la esfera privada y que permite, por tanto, una vinculación del sujeto a la comunidad más amplia (Garretón, 2014; Delamaza, 2002; Olvera, 1999). Como se observará en la siguiente entrevista, aún es posible avanzar en la comprensión de la sociedad civil, desmitificando ciertos usos del concepto y otorgándole una mayor flexibilidad para el entendimiento de las actuales sociedades nacionales.

AR: Muchas gracias Profesor por este tiempo que ha cedido de su apretada agenda. En Chile se ha seguido con entusiasmo su trabajo, en particular por aquellos interesados en las materias de ciudadanía, construcción democrática y sociedad civil. Como una primera cuestión a desarrollar, podría decirnos: ¿Cuáles son los proyectos de investigación que actualmente le demandan más atención? 
AO: Actualmente tengo dos proyectos principales. Uno se refiere al estudio del acceso a la justicia para los mexicanos, con especial énfasis en el acceso a la justicia para mujeres y a la justicia laboral en México, tema central también para otros países de América Latina, pero no en Chile. En la mayoría de los países de América Latina la falta de funcionamiento del sistema de justicia es un dato estructural, sistémico y esto ha conducido a profundizar las desigualdades socioeconómicas y sobre todo, a agravar la desigualdad política, es decir, el acceso a y el ejercicio de derechos. Este tema es poco entendido en México, en donde el acceso a la justicia nunca se entendió como un servicio fundamental del Estado, sino como una especie de aparato burocrático secundario cuyo acceso era básicamente mercantil, es decir, dependía de cuánto dinero tenía el ciudadano que caía en sus garras. La justicia se convirtió en un aparato institucional que extraía rentas a los pobres, y también a los ricos; un sistema cuya corrupción estructural ha determinado que el acceso al mismo sea segmentado y desigual. Éste es un estudio que, a diferencia de otros en este campo, es muy etnográfico: procedí a través de entrevistas a víctimas, a personas que llevan sus casos, entrevisté a policías, agentes del Ministerio Público y jueces, y he entrevistado más recientemente a los colectivos de víctimas de desaparición forzada. En México tenemos un problema gravísimo de asesinatos vinculados al crimen organizado, pero también de desapariciones forzadas, la mitad de las cuales tienen que ver con la acción de los agentes del Estado, la otra mitad con la acción de los criminales. Este es un problema no solo de México, sino de Centroamérica, Colombia, Brasil y Venezuela, que es el peor caso de América Latina en ese aspecto de la violencia generalizada. Tanto el crimen organizado como las fuerzas de seguridad en su combate a aquél están victimizando básicamente a los pobres. Este es el tema que desarrollo recientemente y el otro es la continuidad de investigaciones anteriores que se refieren a explicar por qué en México no logra democratizarse, no sólo en la vida política, sino en la social, es decir, por qué las relaciones autoritarias siguen marcando la vida pública y por qué sobrevive el viejo régimen a pesar de la alternancia electoral. Para este fin sigo desarrollando una reflexión comparativa con otros países de América Latina.

AR: ¿Cómo cree usted que ha evolucionado el campo de estudio de la sociedad civil hasta el día de hoy?

AO: La idea de sociedad civil generó grandes expectativas. Las tres décadas pasadas fueron las de la gran ilusión de la sociedad civil. Fue la época de las transiciones a la democracia en América Latina y del ascenso de las luchas antiautoritarias en el mundo socialista. A partir de la caída del muro de Berlín y de la relativa consolidación de las democracias electorales en América Latina, el concepto tendió a desdibujarse, en parte porque estaba demasiado cargado de un contenido normativo hasta cierto punto errado, producto de la herencia liberal de la idea de sociedad civil. Primero, se asumió que la sociedad civil era, como dijera mi colega Evelina Dagnino, una especie 
de campo de la virtud, el campo del heroísmo ciudadano frente a un Estado autoritario; se construyó una imagen binaria: Estado malo vs. sociedad buena. La sociedad civil representaba la defensa de la libertad y de los derechos de ciudadanía. Pero después de las transiciones y de la caída del muro de Berlín, la pluralidad política, cultural y valorativa que portan los actores de la sociedad civil quedó más clara que nunca. El caso de Polonia, por ejemplo, demostró cómo una vez sustituido el sistema autoritario, la sociedad civil se fragmenta en los múltiples grupos que en su interior conviven. Es decir, emerge la pluralidad política y cultural de la sociedad civil, y se produce también un rápido proceso de translación de cuadros desde lo civil a lo político, de conformación de partidos políticos, y la consiguiente transformación de los que eran líderes civiles en líderes políticos. Esto mismo sucede parcialmente en otros países de América Latina, en los que un sector importante de los activistas civiles, una vez que se llega a la democracia electoral, se incorporan a los gobiernos legítimamente electos, perdiéndose el liderazgo civil previamente creado, y se confirma así la hipótesis adelantada ya desde los tiempos de O'Donnell y Schmitter en los estudios de las transiciones a la democracia, en el sentido de que la sociedad civil era importantísima en la fase de la transición para movilizar a la sociedad, pero que una vez consumada la democratización electoral, la sociedad civil perdería su protagonismo y centralidad. Se ha confirmado que eso es así, pero no por lo que pensaban los transitólogos: que el campo de la democracia requiere ineludiblemente de partidos políticos, sino porque los políticos profesionales, que no los partidos, en efecto son necesarios.

No nos habíamos dado cuenta de que la sociedad civil en realidad contiene en su propio seno todos los conflictos propios de la sociedad, que ella misma es un campo de conflicto. Creo que esto ha sido difícil de entender hasta la fecha. Precisamente porque la sociedad civil refleja en su interior los conflictos propios de la sociedad, es imposible que se encuentren puntos en común de todos los componentes de la misma, excepto en las condiciones limite, en los momentos de quiebre, en los momentos en que un régimen empieza a colapsar. El punto común que une a los distintos actores de la sociedad civil es la defensa de los derechos humanos, porque todos los actores deben tener protegidos sus derechos, pero más allá de ese punto común -que sin embargo contiene elementos en debate-, emerge la pluralidad. Son miembros de la sociedad civil los que defienden el derecho de las mujeres a tomar decisiones sobre su cuerpo, como lo son los antiabortistas, por poner un ejemplo radical. Hay un debate entre ellos respecto si es un derecho de las mujeres el de abortar o no lo es. También lo es si los migrantes tienen derechos humanos por el solo hecho de ser seres humanos o no los tienen porque son extranjeros y, por tanto, no merecen la protección de las leyes nacionales. Hay debates internos a la sociedad civil sobre la extensión de los derechos humanos. Creo que estamos hoy en un periodo en el cual la idea de sociedad civil perdió ese sentido valorativo homogeneizante, positivo y heroico que 
se le atribuyó, y tenemos una comprensión postliberal más fina que nos muestra que mucho de la construcción de los imaginarios políticos y sociales tiene que ver con la resolución de conflictos dentro de y para la sociedad civil.

AR: ¿Qué otras tendencias podrían dar cuenta dela sociedad civil latinoamericana?

AO: Precisamente por la pluralidad que he mencionado, la sociedad civil no actúa en una sola dirección, por lo que la resolución de sus conflictos exige no sólo la democracia electoral, sino la existencia de condiciones políticas y sociales que canalicen los conflictos intrínsecos a la sociedad en una forma civilizada, que proteja los derechos de todos. ¿A qué me refiero? Quiero decir que es fundamental entender que los conflictos distributivos y valorativos de una sociedad se resuelven no sólo en el Estado, sino dentro de la sociedad civil. Los conflictos religiosos, los conflictos generacionales, los laborales, todos los conflictos de la sociedad necesitan formas de solución civilizada. Si no, no podemos avanzar, pues la democracia por sí misma no resuelve ningún problema social fundamental, es tan sólo una forma de resolver problemas políticos y canalizar problemas sociales de una manera civilizada, pero eso no presupone que el campo de la política electoral pueda resolver los problemas intrínsecos a la vida social. Debemos entender que los conflictos de la sociedad necesitan espacios de expresión y nuevas formas de canalización del conflicto social a lo político. La intermediación entre la sociedad y la política no puede ser resuelta solamente por los partidos políticos y, al aceptar esto, nos encontramos ante un campo no resuelto respecto a cuales pueden ser esas mediaciones. Entendemos, sin embargo, que se necesita un Estado de derecho, la construcción de un verdadero régimen democrático.

AR: En ese mismo sentido, la propuesta teórica de comprensión de la sociedad civil, separada del Estado y separada del mercado presenta ciertos problemas, empieza a crujir la estructura teórica habermasiana aplicada al estudio de la sociedad civil porque es muy difícil entender la sociedad civil sin relaciones permanentes, conflictivas con el Estado y con el mercado ¿Cuál es su apreciación respecto de estas relaciones entre la sociedad civil, el Estado y el mercado?

AO: La social civil en la perspectiva habermasiana, tal como fue desarrollada por Cohen y Arato, supone la existencia de dos elementos centrales: una es la autonomía organizacional y política de la sociedad civil respecto al Estado y otra es la autolimitación, es decir, el principio de que los actores sociales no pueden aspirar a ocupar el poder político por sí mismos. De hecho, esta teorización producida por Andrew Arato y Jean Cohen en su clásico libro Teoría Política y Sociedad Civil (200o), recogía la autocomprensión de los propios actores sociales que luchaban contra el totalitarismo en Europa Oriental y contra las dictaduras en América Latina en la primera mitad de los años ochenta.

AO: Ahora nos damos cuenta que esas dos características, autonomía y autolimitación, describen la periferia política de la relación entre la sociedad civil y el Es- 
tado, pero que en realidad, esta caracterización no va al fondo de las cosas, pues la sociedad, el Estado y el mercado no están separados en la práctica social. No podemos tomar la separación liberal de las tres instancias, útil para fines analíticos, como una descripción adecuada de la realidad. Lo central es entender los conflictos. Los conflictos están dados en las relaciones sociales mismas y la autonomía del poder y la autolimitación de la sociedad civil no los resuelven ni los reconocen. Insisto, la sociedad civil es ella misma un campo de conflicto y necesita sus propios mecanismos de procesamiento. Anteriormente habíamos pensado en esa separación Estadosociedad civil de tal forma que habíamos constituido a la sociedad civil en un campo pre - político de la realidad social. En realidad la sociedad civil es un campo político, sólo que no se expresa solamente a través de la política electoral y partidaria, sino tiene otros mecanismos de canalización en las esferas pública y privada, por lo que constituye un campo de lo político en tanto a su interior se procesan conflictos. Y los conflictos implican política. Nada más claro para entender esto que el mundo de las relaciones laborales, en donde se confrontan sindicatos y empresarios, pero ese tipo de relaciones se producen, se multiplican al infinito en múltiples campos debido a la complejidad de las relaciones sociales.

AR: Transitando desde el plano teórico al práctico, para hacer algún análisis de lo que sucede en nuestra región, hemos visto la avanzada de violencia en nuestro país hermano Venezuela y no queríamos dejar fuera una mención por parte suya respecto de este caso en donde la sociedad civil parece manifestarse de manera inusual, ¿Qué lectura hace usted respecto de esta situación?

AO: Es muy complejo el tema, pero digamos que el caso de Venezuela lo que expresa en este momento es una crisis de régimen. Se trata de un régimen híbrido que, respetando las formalidades democráticas, había logrado alcanzar una hegemonía política nacional con enorme apoyo popular, pero este apoyo popular nunca logró ser orgánico, en el sentido de haber conducido a la organización de la sociedad para participar realmente en la política cotidiana. Había un apoyo de masas inorgánico, en donde el vínculo entre la sociedad y el Estado estaba mediado por un líder, Hugo Chávez. Este carácter inorgánico implica que la relación política entre el Estado y la sociedad sea más bien plebiscitaria y ese orden solamente puede mantenerse mientras estas mayorías sienten que obtienen algún beneficio, algún tipo de reconocimiento efectivo por parte del Estado. Este es el clásico vínculo populista. Ese ciclo terminó con la crisis abierta por la caída del precio del petróleo desde 2013, a la que se sumó el pésimo manejo macroeconómico del gobierno, que condujo a una crisis económica monumental, con la inflación más alta del mundo, la escasez de alimentos y medicinas, la caída en picada del poder adquisitivo y la parálisis de la de por sí escasa industria nacional, todo ello en el que fue el país más rico de América Latina en los años setenta. De manera que el apoyo de masas se debilitó y ahora solamente se produce, 
de manera controlada, a través del partido único, el PSUV, y este aparato tiene un control que se vuelve cada día más específico y menos general. Más específico porque solo puede tener soporte de los beneficiarios que son cada vez menos en número y, los leales que son pocos también y no la mayoría de la población. No obstante, los leales y los que apoyan porque tienen algún beneficio, son una parte de una sociedad civil popular que tuvo sus mejores momentos en la segunda mitad de la década pasada, en la que gozó de altos beneficios materiales, reconocimiento simbólico e integración sin mediación con el Estado. Los otros sectores de la sociedad están en contra de Maduro y recurren a las instituciones democráticas para tratar de lograr que en las urnas se demuestre que son mayoría y que la minoría representa el viejo proyecto chavista, sin que el régimen lo permita. El gobierno de Maduro ha impedido que se realicen elecciones regionales y que se utilice la figura de la revocación de mandato, y organizó la elección fraudulenta e inconstitucional de una asamblea constituyente que de inmediato asumió poderes absolutos. Se trata de un golpe de Estado de facto, la conversión del régimen chavista en una especie de régimen militar por la cantidad de militares que participan en la administración pública y porque el ejército es el último recurso de sostenimiento del régimen. Venezuela se ha tornado en un régimen que, bajo el nombre de socialismo del siglo XXI, del que no hay traza alguna en la práctica, se ha constituido de facto en una dictadura militar disfrazada con un barniz democrático cada vez más tenue. Es una tragedia fuera de tiempo, un proyecto falto de vigencia que no tiene futuro y que conducirá simplemente a incrementar los padecimientos de la sociedad venezolana. Hay que decir, sin embargo, que esto ha sido posible también por la increíble incompetencia política de los líderes de la oposición, como increíble y sin precedente es la incompetencia de Maduro como gestor gubernamental. La oposición, muy dividida y heterogénea, ha tomado pésimas decisiones, ha profundizado las divisiones, y al encono propio del populismo generado por Chávez, ha sumado el suyo propio, el tipo de encono de clase que se produce en un sector de la sociedad civil venezolana. Tenemos hoy en Venezuela un experimento de todo lo que no debe hacerse ni en el campo populista ni en el campo de la oposición. Y ésa es la tragedia.

AR: ¿Qué recomendaciones les daría a los/as investigadores/as de pre y postgrado con interés en el estudio de la sociedad civil y los procesos políticos de democratización al interior de las sociedades latinoamericanas?

AO: Los estudiantes tienen que abandonar toda pretensión de alcanzar una verdad absoluta a partir de los marcos teóricos disponibles. La ciencia política tiene en general un grave déficit sociológico y la sociología un grave déficit politológico. El poder no se puede entender al margen de la conflictividad social y de la agencia humana, y éstas a su vez no se explican si no consideramos las relaciones de poder. La separación disciplinaria en campos de conocimiento distintos produce resultados parciales e incompletos. Las ciencias sociales son especialmente precarias en lo que se refiere 
a sus instrumentos teóricos. De manera que es necesario desarrollar una capacidad crítica y una imaginación sociológica, como dijera hace más de cincuenta años el hoy olvidado C. Wright Mills.

AR: Para finalizar ¿Qué conceptos y enfoques pueden ayudar hoy en día a una comprensión más acabada de la sociedad civil y la movilización de actores sociales?

AO: Todos. Insisto que es imprescindible desarrollar la capacidad de combinar críticamente los más diversos enfoques y métodos. Ante todo, es necesario desarrollar trabajo de campo y hacer etnografía, no porque todos los cientistas sociales y políticos deban tener algo de antropólogos, sino porque no es posible hacer ciencia social sin comprender al otro, al observado que nos observa, sin ponernos en su lugar. Por ello veo con particular preocupación el excesivo gusto por las encuestas, como si los promedios estadísticos reflejaran una forma realmente existente de opinar sobre el mundo. Estamos cada vez más acostumbrados a establecer categorías abstractas, a clasificar y segmentar grupos, mercados y preferencias. Los seres humanos reales son complejos y multidimensionales. Una ciencia social que no parta de este principio no es ciencia social.

AR: Muchas gracias Profesor por su disposición.

\section{Referencias}

Arato, A., \& Cohen, J. (2000). Sociedad civil y teoría política. Fondo de Cultura Económica: México.

Dagnino, E., Olvera, A. \& Panfichi, A. (2006). La Disputa por la Construcción Democrática en América Latina. Fondo de Cultura Económica-CIESAS-UV.

Delamaza, G. (2002). « Sociedad Civil y Construcción de Capital Social en América Latina: ¿Hacia dónde va la investigación? », Polis [En línea], 2 | 2002. DOI: 10.4000/polis.8711 ᄀ

Garretón, M.A. (2014). Las ciencias sociales en la trama de Chile y América Latina. Estudios sobre transformaciones sociopolíticas y movimiento social. LOM

Isunza, E. \& Olvera, A. (2006). Democratización, Rendición de Cuentas y Sociedad Civil: participación ciudadana y control social. CIESAS-UV-M. A. Porrúa.

Olvera, A. (1999). (coord.) La Sociedad Civil: de la Teoría a la Realidad. Colegio de México: México.

Olvera, A. (2003). (coord.) Sociedad Civil, Esfera Pública y Democratización en América Latina. Fondo de Cultura Económica-Universidad Veracruzana: México.

Olvera, A. (2010a). (coord.) La Democratización Frustrada: Limitaciones Institucionales y Colonización Política de las Instituciones Garantes de Derechos y de Participación Ciudadana en México. CIESAS-UV. 
Olvera, A. (2010b). The elusive democracy: Political Parties, Democratic Institutions, and Civil Society in Mexico. En Latin American Research Review, Latin American Studies Association, University Of Pittsburgh Press, USA, Special Issue, Fall 2010, Vol. 45, pp. 79-107.

Olvera, A. (2012). Ciudadanía precaria y crisis de la estatalidad democrática en América Latina: lecciones para México. En Flores, E. \& Cossío J.R. (coordinadores), La perspectiva mexicana en el siglo XXI, Conaculta, Universidad Veracruzana, México.

Olvera, A. (2015). Una mirada a las rebeliones y protestas en América Latina en el arranque del siglo XXI. En Tahar- Chaouch, M. (coordinador) Protestas, conflictos y cambio político en el mundo árabe y en América Latina; Universidad Veracruzana, México.

Olvera, A. (2016). La sociedad civil en México: entorno, actualidad y perspectivas. En Al. Caldera Al. \& Chaguaceda, A (coords.) Democracia en América Latina: entre el ideal utópico y las realidades políticas, Editorial Fontamara, México.

Olvera, A. (2016). Elecciones presidenciales en México 2012: crisis del Estado y restauración precaria. En Mayorga, F. (compilador). Elecciones y legitimidad en América Latina. CLACSO, La Paz, Bolivia.

Olvera, A. (2017). Legitimidad en el movimiento de derechos humanos en México: Trayectoria histórica y retos del presente. En Guillén, D. \& Monsiváis A. (coords.). La legitimidad como desafío democrático. Expectativas públicas, capacidades institucionales y descontentos ciudadanos. Tijuana: El Colegio de la Frontera Norte.

Olvera, A. (2017). Democracia participativa. En Pereda, C. (ed.). Diccionario de Justicia. México: Siglo XXI.

\section{Sobre el autor}

Andrés Roldán Tonioni es Sociólogo, Doctor (c) en Ciencias Sociales, Universidad de Chile. Correo electrónico es roldantonioni.andres@outlook.es 


\title{
CUHSO. CULTURA-HOMBRE-SOCIEDAD
}

Fundada en 1984, la revista CUHSO es una de las publicaciones periódicas más antiguas en ciencias sociales y humanidades del sur de Chile. Con una periodicidad semestral, recibe todo el año trabajos inéditos de las distintas disciplinas de las ciencias sociales y las humanidades especializadas en el estudio y comprensión de la diversidad sociocultural, especialmente de las sociedades latinoamericanas y sus tensiones producto de la herencia colonial, la modernidad y la globalización. En este sentido, la revista valora tanto el rigor como la pluralidad teórica, epistemológica y metodológica de los trabajos.

\author{
EDITOR \\ Matthias Gloël \\ CoOrdinadora EDITORIAL \\ Claudia Campos Letelier \\ CORRECTOR DE ESTILO Y DISEÑADOR \\ Angélica Vera Sagredo \\ TRADUCTOR, CORRECTOR LENGUA INGLESA \\ Aurora Sambolin Santiago \\ Desarrollador de Sistemas \\ Laura Navarro Oliva \\ SITIO WEB \\ cuhso.uct.cl \\ E-MAIL \\ cuhso@uct.cl
}

LICENCIA DE ESTE ARTÍCULO

Creative Commons Atribución Compartir Igual 4.0 Internacional 